\title{
Elliptical Fourier descriptors of leaf outlines: a tool to discriminate among Aquilaria species (Thymelaeaceae).
}

\author{
Arlene López-Sampson ${ }^{1}$, Tony Page ${ }^{2}$ \\ 1 College of Science and Engineering, James Cook University, Cairns, Australia 4878. \\ ${ }^{2}$ Tropical Forests and People Research Centre, University of the Sunshine Coast, Maroochydore, Australia 4558. \\ E-mail: tpage@usc.edu.au
}

Corresponding author. E-mail: arlene.lopezsampson@my.jcu.edu.au

\begin{abstract}
Leaf shape analysis of three Aquilaria species was undertaken using Elliptical Fourier descriptors (EFD) within a research planting in tropical Australia. This approach was taken to develop a method for discriminating the species in the absence of reproductive traits or genetic analysis and as a morphological scale of leaf variation. Leaf outlines were analysed to distinguish variability between species. Principal components analysis and canonical discriminant analysis were applied to group the species based on 25 Fourier descriptors. Four components were required to explain $78 \%$ of the variance and the first and second canonical functions discriminated three groups of species therefore EFD was a useful approach for analysing leaf outline variability and distinguish species in the genus Aquilaria. This approach provides a rapid method that could assist in the species identification in Aquilaria where reproductive traits are absent.
\end{abstract}

Keywords: : Leaf shape, SHAPE, species discrimination, vegetative characters

\section{Introduction}

Aquilaria species occur naturally in eastern India, south China, Southeast Asia, The Philippines and Papua New Guinea with 20 known species (Ding Hou, 1965, Rae, 1991, The Plant List, 2013). Aquilaria species are trees or shrubs and can be found in areas ranging from sea level up to $1000 \mathrm{~m}$ above sea level and in different soil conditions. Aquilaria species produce a fragrant resin known as agarwood with high market demand. Historically this demand has been satisfied through the unfettered harvesting of the species' natural populations. As such many species in the genus have been listed as threatened or endangered (IUCN, 2013). Reforestation with Aquilaria is now being undertaken in producer countries and other tropical areas to meet increasing demand and alleviate harvesting pressures within natural populations. These programmes have relied on different germplasm sources to establish the plantations and plantings may comprise multiple species. Accurate identification of established trees is important to help determine species composition in planted populations. This knowledge can then be incorporated into the management of the plantations based on the biological tolerances and market preferences for certain species.

Leaves, flowers and seeds can be evaluated to identify and classify cultivars or distinguish morphological variations between species (Bruschi, et al., 2003, Campos, et al., 2005, Jones, et al., 2013). In a plantation, identification of juvenile specimens will depend upon vegetative characters and development of simple morphological tools. Elliptical Fourier analysis (EFA) is commonly used in plants to study the shape of different organs (Iwata, et al., 1998, McLellan and Endler, 1998, Williams, et al., 2012, Yoshioka, et al., 2004). EFA involves the orthogonal decomposition of a shape into a series of additive harmonic ellipses, with each Fourier harmonic referred to a different aspect of outline shape of the object of interest (Ferson, et al., 1985, Kuhl and Giardina, 1982). The Fourier coefficients, two per harmonic, describe the size (amplitude) and angular offset associated to the starting point (Haines and Crampton, 2000). These series of Fourier harmonics, called elliptical Fourier descriptors, are then typically subjected to morphometric statistical analysis to reduce dimensionality and discriminate groups based on their shape. Elliptical Fourier descriptors have been shown to discriminate among species and geographical location based on leaf shapes (Adebowale, et al., 2012, Viscosi and Fortini, 2011). EFA is useful when a specimen lacks homologous points (landmarks) such as some plant leaves.

Apart the general description of leaf morphology presented in taxonomic reviews, there is no previous research of leaf 
shape in Aquilaria genus. Aquilaria species identification relies mostly on reproductive characters associated with flowers, fruits and seeds (Kiet, et al., 2005, Peterson, 1997, Rae, 1991). While reproductive characters are useful discriminating traits, they are not available in juvenile trees and in mature specimens their availability is seasonally dependent. In contrast leaves are easy to collect and always available in healthy trees, so defining leaf shape characters that discriminate among species can aid identification in the absence of reproductive traits.

In this study, EFA technique was used to describe variation in leaf outlines for three putative Aquilaria species growing within a research plantation. This morphometric technique was selected because leaves in Aquilaria species generally have a lack of homologous and consistent landmarks; only two landmarks could be drawn: petiole-blade junction and blade length. The aim of this study was to quantify differences in leaf shape between three putative species to determine if it can be used to discriminate among them. The germplasm used to grow Aquilaria came from different localities of Southeast Asia.

\section{Materials and Methods}

Three species of the genus Aquilaria growing in a research plantation close to Innisfail, Queensland, Australia $\left(17.52^{\circ} \mathrm{S}\right.$, $145.02^{\circ} \mathrm{E}$ ) were identified using vegetative and reproductive characters. In the plantation, $A$. baillonii is defined by its robust trunk and expanded paraboloid crown-shape. Leaves are oval to ovate in shape with mucronate apices, base obtuse to rounded and pinnate leaf venation. Leaf size 13-15 (-17) cm by 5.3$6.5 \mathrm{~cm}$. The stigma is regularly lobed; fruits ovoid (egg shaped) with widest axis at the base. Seeds are dark brown $(11.76 \mathrm{~mm}$ long) with a long funicle aril dark orange ca. $15 \mathrm{~mm}$. Aquilaria crassna trunk is straight and canopy is spheroid in shape and dense and often exhibits pronounced branching at lower height along trunk and branches plagiotropic. Leaves are dark green, smaller, elliptical with acuminate apices. Leaf size 8-9.5 (12.5) $\mathrm{cm}$ by $3.6-6.6 \mathrm{~cm}$. Flowers are yellow. Stigma is capitate; fruits are rounded (spherical) with calyx visibly loose. Seeds are dark brown with a long funicle aril brownish. Aquilaria subintegra has a distinctive tapering trunk with visible buttressing. The canopy is a fattened cone in shape with very light branching. Leaves are oblong in shape, base cuneate to obtuse. Leaf size (14) $19-27.5 \mathrm{~cm}$ by (5) 7-10.5 cm. Flowers are smaller than A. crassna, A. baillonii, with a distinctive sweet-bouquet aroma, white in colour. Fruits are elliptical. Seeds are dark brown and the long funicle aril dark reddish. A thorough botanical description of the three Aquilaria species can be found in Kiet, et al. (2005), Peterson (1997) and Rae (1991).

\section{Leaf collection}

A total of 320 leaves were sampled from three putative species. For each species 10 to 11 trees were sampled. All trees selected for this study were grown under full sunlight with no supplemental water, and were subject to standard agronomic practices (fertilization, weed and pest control, and pruning). Leaves were harvested from trees of 4.7 years ( 56 months) of age. On each tree 10 fully expanded mature leaves were harvested from a single branch sun-exposed located north of the lower crown (up to $3 \mathrm{~m}$ ). Only leaves free from insect or mechanical damage were included in the samples. Leaves that were not fully developed were not included in the sample. Each collected leaf was scanned with the upper (adaxial) surface using an EPSON V370 with a resolution of $300 \mathrm{dpi}$.

Elliptical Fourier descriptors and statistical analysis Leaf images were processed with two different image processing and analysis software (SHAPE Hiroyoshi Iwata, http://lbm. ab.a.u-tokyo.ac.jp/ iwata/shape/ and ImageJ 1.49n - Wayne Rasband National Institute of Health, USA). The SHAPE software was used to graph the variations of leaf outlines accounted for by each PCA. This function was not available in IMAGE J at the time when this study was carried out. Before images were loaded in ImageJ and SHAPE, they were adjusted with image editing software (Paint.net) to ensure continuous colour along the margin of the leaf. A total of 25 harmonics were used per leaf to estimate the EFDs on both software. This number of harmonics were enough to capture/feature the shape of the leaf outline. In ImageJ images were converted to a black and white image, and then leaves were delineated using the ROI (region of interest) manager to indicate the objects to be computed. The elliptic Fourier analysis was performed using the plugin Elliptic Fourier Descriptor (EFD) (Boudier and Tuppe, 2009). EFD coefficients were then exported to a spreadsheet and averaged per individual to overcome variability within individuals. Averaged EFDs were used to perform a canonical discriminant analysis (Ida function contained in R software) to find linear combinations of the original variables that could give best separation between species. In SHAPE a principal component analysis of the EFDs coefficients was performed using the variance-covariance matrix of the coefficients and the software returned the PCA scores for each object. The variations in shape that can be accounted for by each PCA were obtained and graphed using PrinPrint tool/application from SHAPE.

\section{Results}

The PCA analysis of the EFDs demonstrated that for Aquilaria species the use of Elliptical Fourier analysis helped to differentiate species based on leaf outlines/shape. Four components were required to explain $78 \%$ of the variance (Table 1). The combined variation contained within the first and second principal components accounted for $58 \%$ of total variation in leaf outline/shape. The effect of shape by each PC is illustrated in Figure 1. PC1 describes the widening along the length of the leaf and the length to wide aspect of the leaf and captured the range of leaf shape found in the three species; PC2 is capturing the variation in leaf shape from lanceolate to oblanceolate. PC3 
and PC4 explain the variation in the apices and reflect the outline of the leaf. PC5 is related to the general shape of the leaf (Figure 1). The canonical analysis indicated that groups of species can be separated using leaf outlines, $73 \%$ of separation is achieved with the first canonical function and the remaining $27 \%$ is explained by the second canonical function. The first and second discriminant functions plotted in Figure 2 show that these two functions help in the differentiation of the three Aquilaria species. A. baillonii is clearly separated from A. subintegra and $A$. crassna by the second discriminant function.

Table 1

Importance of the first five principal components explaining the variability of the data.

\begin{tabular}{llll}
\hline $\begin{array}{l}\text { Principal } \\
\text { Components }\end{array}$ & Eigenvalue & $\begin{array}{l}\text { Proportion per } \\
\text { PC }^{\mathrm{a}}\end{array}$ & $\begin{array}{l}\text { Cumulative proportion PC } \\
(\%)\end{array}$ \\
\hline PC1 & $3.20 \mathrm{E}-03$ & 44.60 & 44.60 \\
PC2 & $9.53 \mathrm{E}-04$ & 13.28 & 57.89 \\
PC3 & $7.90 \mathrm{E}-04$ & 11.02 & 68.91 \\
PC4 & $6.64 \mathrm{E}-04$ & 9.26 & 78.17 \\
PC5 & $4.13 \mathrm{E}-04$ & 5.75 & 83.93
\end{tabular}

PC: principal component

$-2 S . D$

Mean

+2 S. D.

PC1

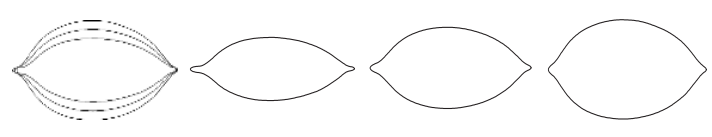

PC2

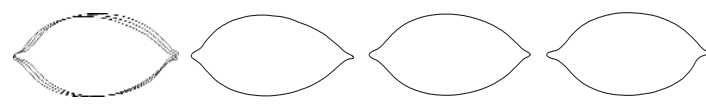

PC3

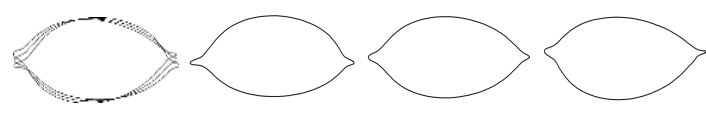

PC4

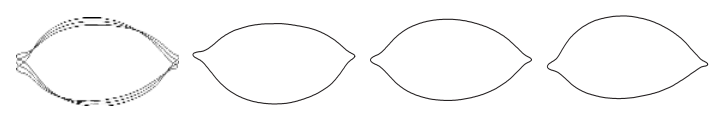

PC5

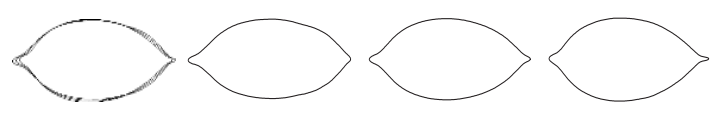

Figure 1

Leaf shape variation of three Aquilaria spp. accounted for by the five first principal components returned from SHAPE.

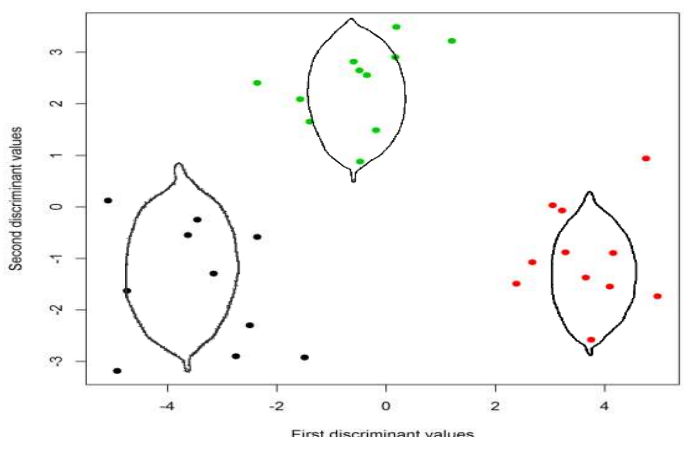

Figure 2

Canonical discriminant analysis (CDA) of the elliptical Fourier descriptors and three Aquilaria species (red circle=A. crassna, green circle $=A$. subintegra, black circle $=A$. baillonii). Leaf outlines representing the three Aquilaria spp are superimposed in the groups formed by the CDA analysis.

\section{Discussion}

Morphological studies are a reliable method used for characterisation and generation of information about systematic and ecology (McLellan and Endler, 1998). The analysis of the entire outline of an object using Fourier analysis can be applied to simple shapes such as ovals to more complex object such as irregular leaf outlines (Adebowale, et al., 2012, McLellan and Endler, 1998, Viscosi and Fortini, 2011). This study demonstrated that mathematical interpretation of leaf outlines using elliptical Fourier descriptors can be used as a tool to differentiate individuals at species level in the genus Aquilaria in young plantations. However, the three putative species were first identified based on other characters mainly flower and form characteristics. Validation studies that include a more extensive range of material (leaves) are required to determine whether this approach can be used to differentiate other species of the genus occurring in both natural areas and plantations in the area of natural distribution. The use and selection of a particular morphometric method for analysing leaf shape is important as each available method considers different aspects of the leaf shape leading to biased results (Chitwood and Sinha, 2016). In this study, elliptical Fourier descriptors is a simple technique that can support identification of Aquilaria species, when reproductive and other diagnostic characters are absent and studies at molecular level are out of the reach of the breeder.

The analysis of outlines to describe shapes and reconstruct images is advantageous when landmark-based analysis is not suitable to perform. McLellan and Endler (1998) suggest that elliptical Fourier analysis provides accurate reconstruction of the outline of complex object and can explain the overall complexity of the shape. Other studies have reported the use of leaf shape description to group species (Andrade, et al., 2008, Jensen, et al., 2002, Viscosi and Fortini, 2011). Jensen, et al. (2002) analysing leaves outline of two Acer species and their 
hybrid were able to distinguish two core groups (species) but it was not possible to differentiate their hybrid. Viscosi and Fortini (2011) found that elliptical Fourier analysis was a useful technique for analysing the leaf shape of three oak species and a valuable tool that could discriminate species in the subgenus Quercus.

Elliptical Fourier descriptors of the leaf outline of Aquilaria species provided a rapid method to study shape/outline variability and identify species in the genus Aquilaria. The leaf outline methodology is a fast and convenient technique that may support national authorities and growers of Aquilaria in the identification of individuals in the genus Aquilaria providing certainty to the owners of the material planted and management strategy.

\section{Acknowledgements}

This research was conducted in Wescorp Argawood research plantation. A.L.S. was in receipt of an Australia Award Postgraduate Scholarship (AusAID).

\section{References}

Adebowale, A., A. Nicholas, J. Lamb and Y. Naidoo (2012) Elliptic Fourier analysis of leaf shape in southern African Strychnos section Densiflorae (Loganiaceae). Botanical Journal of the Linnean Society 170: 542-553. https://doi.org/10.1111/j.1095-8339.2012.01308.x

Andrade, I.M., S.J. Mayo, D. Kirkup and C. Van Den Berg (2008) Comparative morphology of populations of Monstera Adans. (Araceae) from natural forest fragments in northeast Brazil using elliptic Fourier analysis of leaf outlines. Kew Bulletin 63: 193-211. https://doi.org/10.1007/s12225-008-9032-z

Boudier, T. and B. Tuppe (2009) Fourier Shape Analysis. Available at $<\underline{\mathrm{http}} / / / \mathrm{im}-$ agejdocu.tudor.lu/doku.php?id=plugin:analysis:fourier shape analysis:start> [cited 20-03-2015]

Bruschi, P., G.G. Vendramin, F. Bussotti and P. Grossoni (2003) Morphological and molecular diversity among Italian populations of Quercus petraea (Fagaceae). Annals of Botany 91: 707-716. https://doi.org/10.1093/aob/mcg075

Campos, E.T., M.A.G. Espinosa, M.L. Warburton, A.S. Varela and Á.V. Monter (2005) Characterization of mandarin (Citrus spp.) using morphological and AFLP markers. Interciencia 30: 687-693.

Chitwood, D.H. and N.R. Sinha (2016) Evolutionary and environmental forces sculpting leaf development. Current Biology 26: R297-R306. https://doi.org/10.1016/j.cub.2016.02.033.

Ding Hou, L. (1965) Flora Malesiana, Netherlands.

Ferson, S., F.J. Rohlf and R. Koehn (1985) Measuring shape variation of two dimensional outlines. Systematic Zoology 34: 59-68. https://doi.org/10.1093/sysbio/34.1.59

Haines, J. and J.S. Crampton (2000) Improvements to the method of Fourier shape analysis as applied in morphometric studies. Palaeontology 43: 765783. https://doi.org/10.1111/1475-4983.00148

IUCN (2013) IUCN Red list of threatened species version 2013.1. Available at $<$ http://www.iucnredlist.org/search> [cited 02-09-2013]

Iwata, H., S. Niikura, S. Matsuura and Y. Takano (1998) Evaluation of variation of root shape of Japanese radish (Raphanus sativus L.) based on image analysis using elliptic Fourier descriptors. Euphytica 102: 143-149. https://doi.org/10.1023/a:1018392531226

Jensen, R.J., K.M. Ciofani and L.C. Miramontes (2002) Lines, outlines, and landmarks: morphometric analyses of leaves of Acer rubrum, Acer saccharinum
(Aceraceae) and their hybrid. Taxon 51: 475-492. https://doi.org/10.2307/1554860

Jones, A.M.P., S. Murch, J. Wiseman and D. Ragone (2013) Morphological diversity in breadfruit (Artocarpus, Moraceae): insights into domestication, conservation, and cultivar identification. Genet Resour Crop Evol 60: 175-192. https://doi.org/10.1007/s10722-012-9824-8

Kiet, L.C., P.J.A. Kessler and M. Eurlings (2005) A new species of Aquilaria (Thymelaeaceae) from Vietnam. Blumea - Biodiversity, Evolution and Biogeography of Plants 50: 135-141. https://doi.org/10.3767/000651905x623319

Kuhl, F.P. and C.R. Giardina (1982) Elliptic Fourier features of a closed contour. Computer Graphics and Image Processing 18: 236-258. https://doi.org/10.1016/0146-664x(82)90034-x

McLellan, T. and J.A. Endler (1998) The realative success of some methods for measuring and describing the shape of complex objects. Systematic Biology 47: 264-281. https://doi.org/10.1080/106351598260914

Peterson, B.-H. (1997) Thymelaeaceae. Flora of Thailand 6: 226-245.

Rae, S.J. (1991) Thymelaeaceae. Flora of Bhutan 2: 208-213.

The Plant List (2013) Version 1.1. Available at <http://www.theplantlist.org/ tpl1.1> [cited 07/04/2017]

Viscosi, V. and P. Fortini (2011) Leaf shape variation and differentiation in three sympatric white oak species revealed by elliptic Fourier analysis. Nordic Journal of Botany 29: 632-640 https://doi.org/10.1111/j.1756-1051.2011.01098.x

Williams, K., J. Munkvold and M. Sorrells (2012) Comparison of digital image analysis using elliptic Fourier descriptors and major dimensions to phenotype seed shape in hexaploid wheat (Triticum aestivum L.). Euphytica 190: 99-116. https://doi.org/10.1007/s10681-012-0783-0

Yoshioka, Y., H. Iwata, R. Ohsawa and S. Ninomiya (2004) Analysis of petal shape variation of Primula sieboldii by elliptic Fourier descriptors and principal component analysis. Annals of Botany 94: 657-664. https://doi.org/10.1093/aob/mch190 